\section{OBTAINING AND TRANSFERRING ADEQUATE EVIDENCE FOR INTEGRATED CARE}

Liset Grooten. Vrije Universiteit Brussel, Brussel, Belgium

\subsection{6/bmjebm-2019-EBMLive.69}

Obtaining and transferring adequate evidence for integrated care. Problem: In many countries health and social care are too often fragmented and subsequently result in deficiencies in service delivery, causing low responsiveness of the health system, low satisfaction of patients with health services, low job-satisfaction, suboptimal health outcomes, and inefficient use of resources. Integrated care initiatives are being developed around the world to drive forward transformation of health systems to achieve accessible, quality, effective and sustainable health care. However, despite attempts by the Evidence-based medicine movement to emphasize the importance of using evidence to inform healthcare practice, the quality of research providing evidence supporting integrated care initiatives remains unsatisfactorily. This is partly caused by the use of traditional evaluation efforts that focus on outcomes and illustrate an oversimplification of the environment and the complex initiatives. The linkage between changes in services and service outcomes is problematic because most patient or service user outcomes do not emerge from linear cause and effect chains. Current methods and measures of quality in health care provide limited information about the effectiveness of complex interventions within uncontrolled, context-rich settings, and do not clarify the underlying mechanisms governing the components of integrated care. Objective: To achieve a successful transformation towards more integrated care systems, adequate insights are needed into what factors contribute to the progress and success of integrated care initiatives. Possible solution: What is needed are multi-method approaches to evaluation, such as realist research (RR), which captures the complexity of integrated care as reflected in its multiple components and dynamic nature with a view to understanding what works, for whom, and under what conditions. Since the use of comprehensive evaluation approaches, such as RR, is relatively new to scientist in the field of integrated care, it is important to raise awareness on the shortcomings of current evaluation approaches and the need for new comprehensive evaluation approaches. Not only among scientists, but also among doctors, policymakers, and the public. Next to obtaining adequate evidence on integrated care initiatives, it is important to transfer the findings to a wider audience to achieve a wide scale change in health systems. In order not to make the same mistake by having a too narrow perspective on the process of knowledge transfer, as is currently the case, it is important to regard the transfer process as a complex process of interaction and collaboration. Understanding context and the diverse types of knowledge have been observed to be more and more important in affecting behaviour change. Furthermore, evaluation of these complex knowledge translation interventions must be part of the knowledge transfer process, to collect lessons to understand what works, for whom, and under what conditions.

\section{2

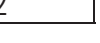

THE ROLE OF THE RESEARCH ADVISORY COUNCIL (RAC) IN BRIDGING THE EVIDENCE-TO-POLICY GAP IN MATERNAL AND CHILD HEALTH IN ETHIOPIA

${ }^{1}$ Mirkuzie Woldie, ${ }^{2}$ Bereket Yakob, ${ }^{3}$ Peter Berman, ${ }^{2}$ Sarah Herlburt. ${ }^{1}$ Federal Ministry of Health, Addis Ababa, Ethiopia; ${ }^{2}$ Harvard School of Public Health, Addis Ababa, Ethiopia; ${ }^{3}$ University of British Colombia, Vancouver, Canada

\subsection{6/bmjebm-2019-EBMLive.70}

Objectives To report on the experiences and challenges of the Research Advisory Council (RAC) of the Maternal and Child Health Directorate (MCHD) at the Federal Ministry of Health, Ethiopia.

Method Review of products (e.g. policy briefs, technical reports, systematic reviews), meeting minutes and terms of reference of the RAC was supplemented with interview of key persons in the establishment and operation of the advisory council.

Results The RAC is composed of university researchers, partner organizations and staff from the Directorate. We found that the RAC regularly (4-6 times per year) meets to consider policy relevant questions from the MCHD. The maternal thematic group of the RAC produced a technical report based on a systematic review which the group conducted. This report was used by the MCHD to make the decision of including uterine balloon tamponade (UBT) and Non-pneumatic antishock garment (NASG) in the guideline for the management of PPH. The child health thematic group led an implementation research (IR) to test the feasibility and acceptability of treatment of young infants with possible serious bacterial infection (PBSI) by community health workers. This led to the inclusion of this approach in the national guideline for management of sick infants. Its voluntary nature and dependence on external funding were the key challenges.

Conclusions The RAC has proved to be instrumental in bridging the disconnect between those who produce evidence and those expected to use it. Planned efforts to integrate the operation and funding of the RAC in the regular annual plan of the Directorate is recommended.

\section{2DSEARCH: FACILITATING REPRODUCIBLE AND VALID SEARCHING IN EVIDENCE SYNTHESIS}

${ }^{1}$ Tony Russell-Rose, ${ }^{2}$ Farhad Shokraneh. ' UXLabs, Guildford, UK; ${ }^{2}$ Cochrane Schizophrenia Group, Institute of Mental Health, Division of Psychiatry and Applied Psychology, School of Medicine, University of Nottingham, Nottingham, UK

\subsection{6/bmjebm-2019-EBMLive.71}

Objectives Search is the first stage in evidence synthesis, and the use of a valid and reproducible search strategy forms the foundation of the systematic review process. However, the key steps of developing, validating, visualising, saving, sharing, peer-reviewing, and translating search strategies (to the syntax of other interfaces) are often fragmented across a variety of unconnected, non-interoperable platforms. We present an open-access platform that offers a unified approach to structured searching which promotes replicable methods and reproducible results. 